\title{
Intuitionistic fuzzy evaluation of artificial neural network model
}

\author{
Todor Petkov ${ }^{1}$, Veselina Bureva ${ }^{2}$ and Stanislav Popov ${ }^{3}$ \\ 1 "Prof. Dr. Assen Zlatarov" University \\ 1 "Prof. Yakimov" Blvd, Burgas-8010, Bulgaria \\ e-mail: todor_petkov@btu.bg \\ 2 "Prof. Dr. Assen Zlatarov" University \\ 1 "Prof. Yakimov" Blvd, Burgas-8010, Bulgaria \\ e-mail: vbureva@btu.bg \\ 3 "Prof. Dr. Assen Zlatarov" University \\ 1 "Prof. Yakimov" Blvd, Burgas-8010, Bulgaria \\ e-mail: stani_popov@yahoo.com
}

Received: 17 July 2021

Accepted: 20 November 2021

\begin{abstract}
In this paper a method that evaluates a trained artificial neural network is presented. The learning type of an artificial neural network is supervised learning which requires labeled input training vectors. Labeled medical data is provided to train the network, where the labels can either be 1 if the person is alive, or 0 if the person has deceased. The data is divided into training and validation vectors. The validation input vectors are used to evaluate the model and the results are summarized by using intuitionistic fuzzy values.
\end{abstract}

Keywords: Intuitionistic fuzzy evaluation, Intuitionistic fuzzy sets, Neural network. 2020 Mathematics Subject Classification: 03E72.

\section{Introduction}

In the current section, short remarks on artificial neural networks and intuitionistic fuzzy sets are presented. They will be used in the proposed intuitionistic fuzzy evaluation of neural network model. 


\subsection{Artificial neural networks}

Artificial neural networks are inspired by the biological neural network of the human and animal brain [11]. The significance of the biological neural system is in the ability to learn by itself from the surrounding environment. The learning process is a task that scientists are still exploring and the results of their research are implemented into the working models in Artificial Neural Networks (ANN) [1, 2, 12]. The main basic building block of an ANN is the Artificial Neuron shown in Fig. 1.

The artificial neuron tries to represent the work of the biological neuron. Each artificial neuron has one or more inputs and an output; the inputs are the incoming signals from the other neurons and the output is the result from the neuron to the next neuron(s). The inputs to the neuron in Fig. 1 are denoted as $p$ where $p$ is a vector of the incoming signals:

$$
p=\left[p_{1}, p_{2}, \ldots, p_{n}\right],
$$

where $n$ is the number of the incoming signals.

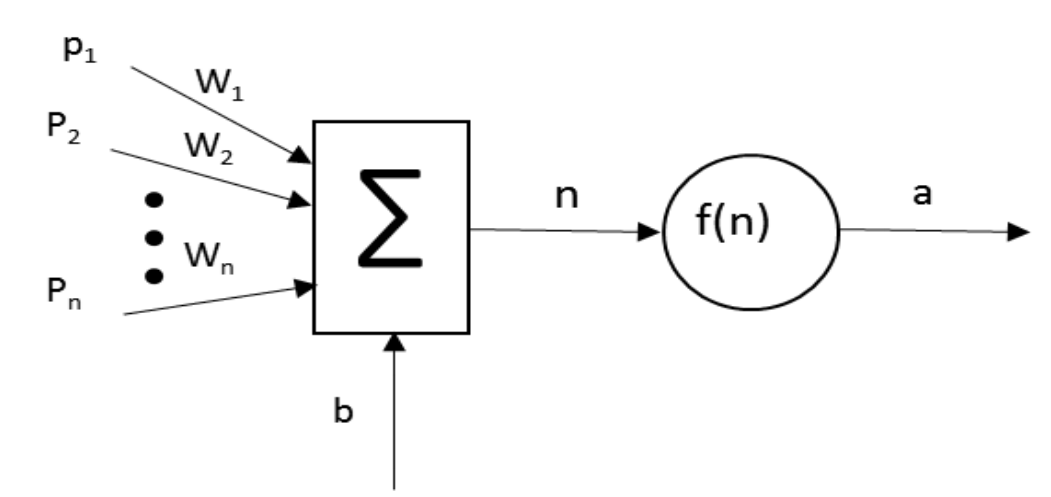

Figure 1. The model of an artificial neuron

The incoming signals are multiplied by the values of the neuron which are called weights

$$
w=\left[w_{1}, w_{2}, \ldots, w_{n}\right]
$$

The multiplication results are summed and the result $n$ is the actual argument of the neuron function $f(n)$. The formula which describes the neuron activation is:

$$
a=f\left(\sum_{i=0}^{n} p_{i} w_{i}\right) .
$$

A single neuron cannot perform complex tasks. In such cases, a multilayer neural network can be used. An example of a multilayer neural network is shown in Fig. 2.

A single layer is composed of neurons where each of them has its own weights and also applies an activation function. A multilayer neural network consists of two or more layers that are fully connected. The output of the previous layer is the input for the next layer. 


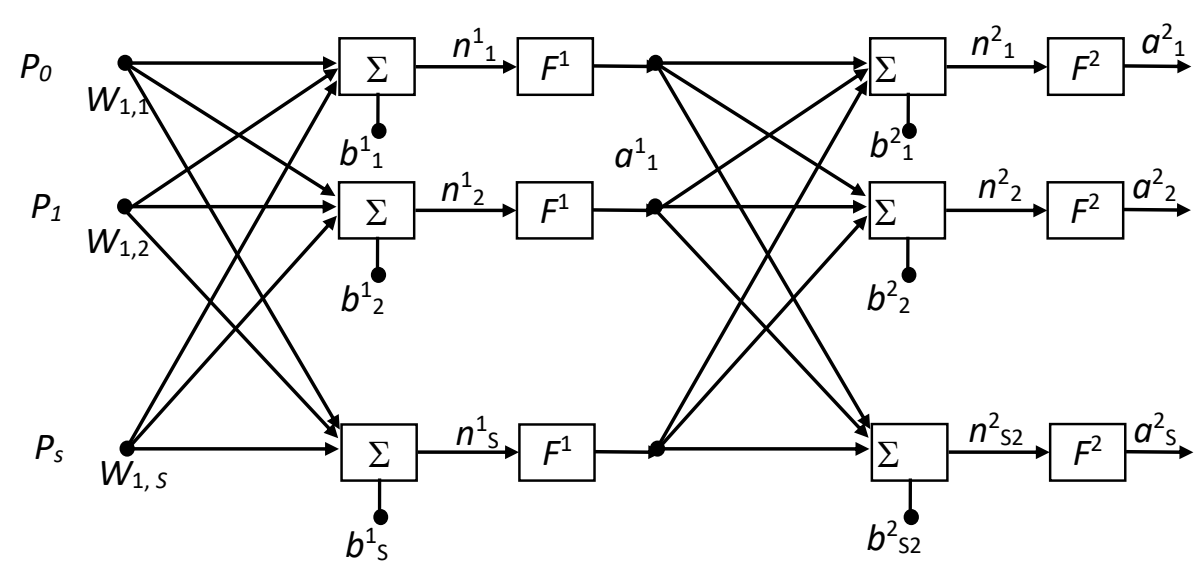

Figure 2. The architecture of a multilayer network

\subsection{Intuitionistic fuzzy sets}

The intuitionistic fuzzy sets are introduced by Atanassov [3] as an extension of the fuzzy sets. The difference between fuzzy sets and intuitionistic fuzzy sets (IFSs) is in the presence of a second function $v_{A}(x)$ defining the non-membership of element $x$ to set $A$, where $\mu_{A}(x) \in[0 ; 1]$, $v_{A}(x) \in[0 ; 1]$, under the condition of $\mu_{A}(x)+v_{A}(x) \in[0 ; 1]$. The IFS is formally presented by:

$$
A=\left\{\left\langle x, \mu_{A}(x), v_{A}(x)\right\rangle \mid x \in E\right\} .
$$

Respectively, and for every $x \in E$ : $0 \leq \mu_{A}(x)+v_{A}(x) \leq 1$. Every ordinary fuzzy set has the form:

$$
A=\left\{\left\langle x, \mu_{A}(x), 1-\mu_{A}(x)\right\rangle \mid x \in E\right\} .
$$

If

$$
\pi_{A}(x)=1-\mu_{A}(x)-v_{A}(x),
$$

then $\pi_{A}(x)$ is the degree of uncertainty of the membership of the element $x \in E$ to the set $A$. In the case of ordinary fuzzy sets, $\pi_{A}(x)=0$ for every $x \in E$, [3-5, 7].

A comparison between elements of any two IFSs, $A$ and $B$, involves pairwise comparisons between their respective elements' degrees of membership and non-membership to both sets. In recent years, the theory of intuitionistic fuzzy sets has been extended with research works in the field of Interval-Valued Intuitionistic Fuzzy Sets [7] and Circular Intuitionistic Fuzzy Sets [5, 8].

\section{Task essentials}

The goal of the paper is to evaluate the model of ANN using intuitionistic fuzzy values. The data that is used to train the model of ANN is taken from $[10,13]$ and it consists of measured values for persons that have heart disease. The aim is to predict whether a person is going to die (marked as 0 ) or live (marked as 1). The input vector for the ANN model consists of 11 values and the explanation of each one of them is the following: Age, Anemia, Creatinine, Diabetes, Ejection fraction, High blood pressure, Serum creatinine, Serum sodium, Sex, Smoking, Time. 
The structure of the ANN model consists of 3 layers of neurons. The first two layers apply Hyperbolic Tangent Sigmoid function:

$$
f(n)=\frac{e^{n}-e^{-n}}{e^{n}+e^{-n}}
$$

The last layer of the ANN applies the linear activation function

$$
f(n)=n .
$$

The ANN has two outputs which describe the following:

- to predict death, the output vector is [1 0$]$;

- to predict if the person is going to live, the output is [0 1 [ 1 .

The general structure of the ANN is shown in Fig. 3. The first layer of the network consists of 21 neurons, the second one consists of 18 neurons and the hyperbolic tangent sigmoid function is applied to both layers. The last layer which applies linear activation function has two neurons.

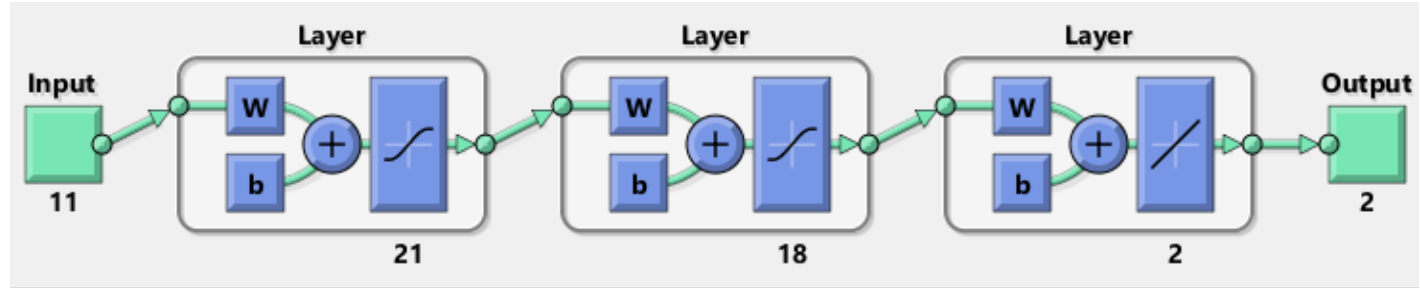

Figure 3. Structure of the ANN

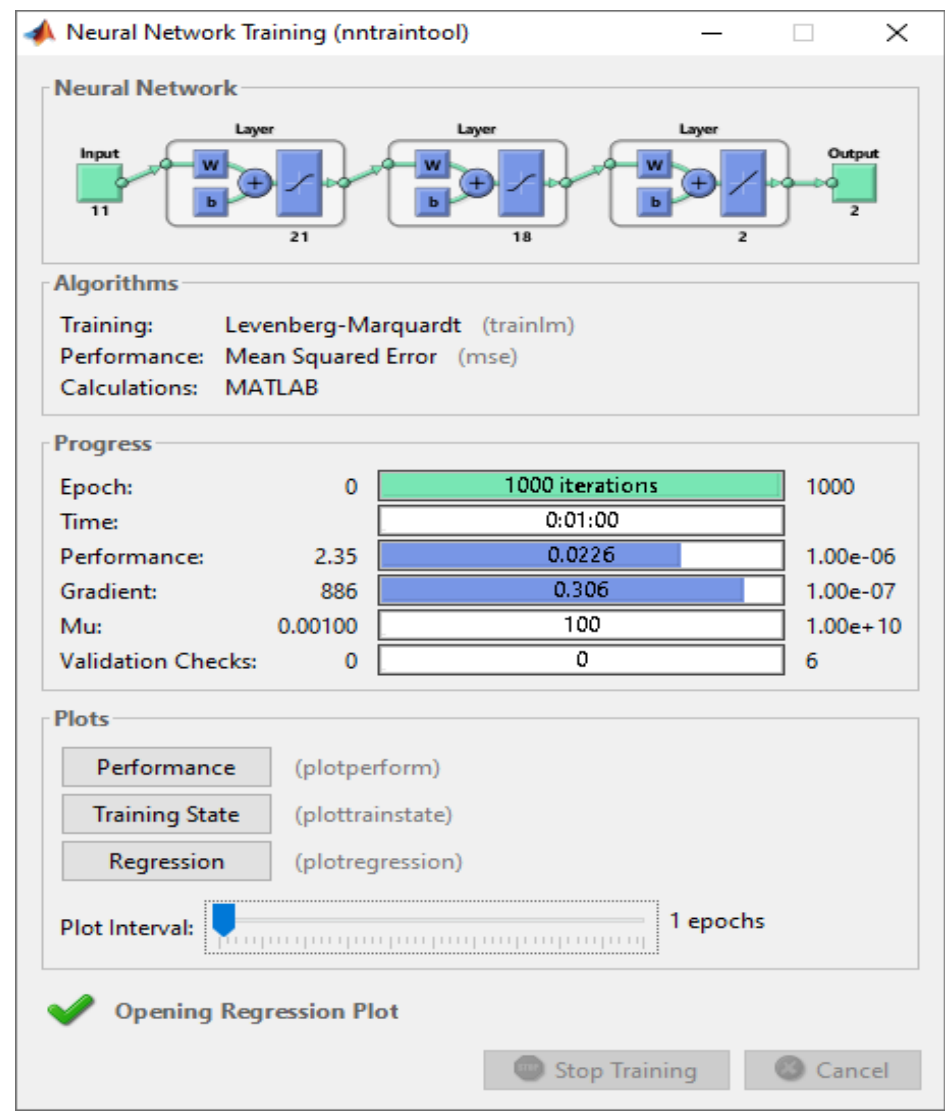

Figure 4. ANN structure and parameters 
The structure of the ANN and its training parameters such as Gradient, Performance and Training Epochs are shown in Fig. 4. The training algorithm of the ANN is Levenberg Marquardt which is responsible to update the network coefficients during the training process.

The regression line shows how well the ANN can predict the correct results. Fig. 5 shows the regression line of the trained ANN. The parameter that determines the prediction success is called $R$ and if the value of that parameter is closer to 1 that means that the ANN can predict each training vector successfully.



Figure 5. Regression line of the ANN

The prediction capabilities of the ANN have to be evaluated. This process is known as ANN validation. In order to validate the ANN, 51 labeled vectors are used to summarize:

- $\quad$ The training vectors are 248.

- The validation vectors (which are not in the training set) are 51.

The expected result for a given input vector from the trained ANN model consists of two values. The evaluation of the ANN is done by using intuitionistic fuzzy (IF) values. In order to evaluate the ANN model, the IF thresholds for $\pi, \mu$ and $v$ have to be chosen.

It was previously explained that for the person that is going to live, the output vector of the ANN has to be [ $\left.\begin{array}{ll}0 & 1\end{array}\right]$. On the contrary, for the death event the expected output from the network has to be $\left[\begin{array}{ll}1 & 0\end{array}\right]$. During the learning trial, the ANN updates its weights and biases and when it is trained, the output values for the given input vector is not necessary to exactly match the labeled ones in our case [0 1 1] or [1 0$]$.

The threshold values from the output of the ANN that are closer to 0 and 1 are:

- $0-0.25 \div 0.25$;

- $1-0.75 \div 1.25$.

The IF values are: 


$$
\begin{cases}0 & 1-\mu \\ 1 & 0-v \\ 0 & 0-\pi \\ 1 & 1-\pi\end{cases}
$$

The degree of membership is estimated by division of [0 1 1]-vectors $l$ predicting that the person is going to live to the total number of validation vectors $s$. The formula has the following form:

$$
\mu=\frac{l}{s}
$$

Therefore,

$$
\mu=\frac{5}{51}=0.1
$$

where the [ $\left.\begin{array}{ll}0 & 1\end{array}\right]$-vectors $l$ predicting that the person is going to live are 5 and the total number of validation vectors are 51.

The degree of uncertainty includes the output vectors [ $\left[\begin{array}{ll}1 & 1\end{array}\right]$ and $\left[\begin{array}{ll}0 & 0\end{array}\right]$ that represent the cases when it is not possible to predict output based on the data - the uncertainty. The formula has the following form:

$$
\pi=1-\mu-\vartheta
$$

Therefore,

$$
\pi=1-0.1-0.35=0.55 .
$$

Fig. 6 shows the graphic of the IF values estimated from the validation set of 51 vectors.

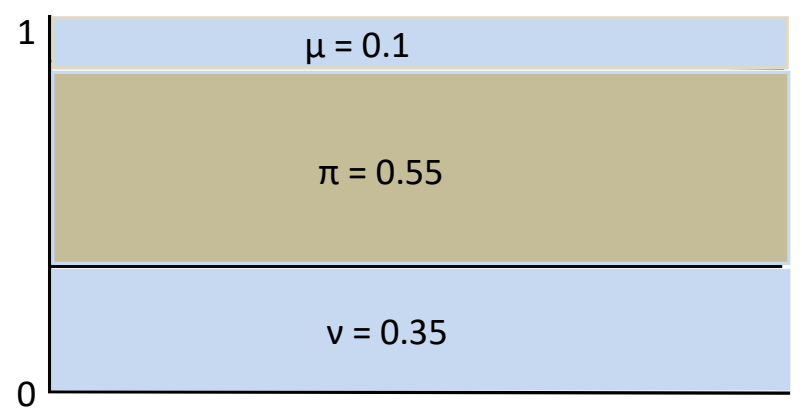

Figure 6. Estimated IF values of the validation set

\section{Conclusion}

In the paper, a method of evaluating an artificial neural network is presented. In order to achieve this goal, medical data is provided along with labelled vectors. The ANN was trained by training vectors and when the process finished, the 51 validation vectors were used to evaluate the process of training. One of the important steps was the choice of the threshold as its choice reflects to the evaluation of the ANN by using IF values. Smaller threshold increases the degree of uncertainty, on the other hand, the bigger threshold increases $v$ and $\mu$ values. It can be concluded 
that using IF values we can successfully evaluate the training of the ANN; if one needs to decrease the $\pi$ value the chosen threshold has to be decreased.

\section{Acknowledgements}

The authors are thankful for the support provided by Project "Analysis and modelling of artificial intelligence algorithms and their application”, No. NIH - 462/2021.

\section{References}

[1] Aggarwal, C. (2018). Neural Networks and Deep Learning: A Textbook. $1^{\text {st }}$ edition, Springer.

[2] Alama, A., Arana-Daniel, N., \& Lopez-Franco, C. (2019). Artificial Neural Network for Engineering Applications. $1^{\text {st }}$ Edition, Elsevier Inc.

[3] Atanassov, K. (2012). On Intuitionistic Fuzzy Sets Theory. Springer, Berlin.

[4] Atanassov, K. (2015). Intuitionistic fuzzy logics as tools for evaluation of Data Mining processes. Knowledge-Based Systems, 80, 122-130.

[5] Atanassov, K. (2020). Circular Intuitionistic Fuzzy Sets. Journal of Intelligent and Fuzzy Systems, 39(5), 5981-5986.

[6] Atanassov, K. (2020). Generalized Nets and Intuitionistic Fuzziness in Data mining . "Prof. Marin Drinov" Publishing House of the Bulgarian Academy of Sciences, Sofia.

[7] Atanassov, K. (2020). Interval-Valued Intuitionistic Fuzzy Sets. Springer, Cham.

[8] Atanassov, K., \& Marinov, E. (2021). Four Distances for Circular Intuitionistic Fuzzy Sets. Mathematics, 9(10), Article No. 1121.

[9] Atanassov, K., Szmidt, E., \& Kacprzyk, J. (2013). On intuitionistic fuzzy pairs. Notes on Intuitionistic Fuzzy Sets, 19(3), 1-13.

[10] Chicco, D., \& Jurman, G. (2020). Machine learning can predict survival of patients with heart failure from serum creatinine and ejection fraction alone. BMC Medical Informatics and Decision Making, 20, Article No. 16.

[11] Hagan, M., Demuth, H., Beale, M., \& De Jesús, O. (2014). Neural Network Design. $2^{\text {nd }}$ Edition, Retrieved from: https://hagan.okstate.edu/NNDesign.pdf. Accessed: 27.09.2021.

[12] Haley, W. (2018). Artificial Intelligence. Grey House Publishing.

[13] Sakhiya, Nayan (2021). Python: Heart Fail: Analysis and Quick-prediction, Retrieved from: https://www.kaggle.com/nayansakhiya/heart-fail-analysis-andquick-prediction/data. Accessed: 27.09.2021. 